\title{
A New Epilepsy Identification Technique Based on VMD, RELIEFF Algorithm and Machine Learning Classification Approach
}

\author{
SK. Ebraheem Khaleelulla ${ }^{1}$, P. Rajesh Kumar ${ }^{2}$ \\ ${ }^{1}$ Research Scholar, Dept. of ECE, AUCE (A), Andhra University, Visakhapatnam, India \\ ${ }^{2}$ Professor and Chairman, BoS, Dept. of ECE, AUCE (A), Andhra University,isakhapatnam, \\ India
}

\begin{abstract}
Epileptic seizure recognition is finished by visual perception of electroencephalography (EEG) signals, which is increasingly touchy to predisposition and tedious. In the vast majority of the past research of epileptic seizure identification experiences unsuitability and low power for handling huge datasets. To take out previously mentioned issues a modernized discovery method is required to help restorative experts. In this paper, another method is proposed to recognize the epilepsy dependent on VMD, RELIEFF algorithm and machine learning approach. To examine the adopted method execution an open EEG dataset is received from university hospital bonn, Germany. The system begins with the VMD, which is utilized to separate the features from every EEG signal. And after that RELIEFF algorithm is used to recognize the best features. At last to sort the ordinary and epilepsy EEG signals a machine learning classification (ANN and $\mathrm{KNN}$ ) approach is utilized. The outcomes exhibit that the adopted method (VMD+RELIEFF+KNN) can accomplish a high accuracy, showing an amazing technique to identification and classification of epileptic seizures.

Keywords: electroencephalogram, variational mode decomposition, RELIEFF.
\end{abstract}

\section{Introduction}

Epilepsy is a neurological issue (any turmoil of sensory system). The reason for most cases, epilepsy happen because of cerebrum damage, strokes or anomalous movement of synapse (i.e., produce multiple times bigger sign than of typical). It causes seizures, loss of mindfulness at times or neuronal movement in the cortex of the cerebrum. Most of epileptic cases are expanding over the world very now and again [1].

Much of the time, epileptic disorders or seizures are controllable with prescription (hostile to epileptic) in about $70 \%$ of cases or in some cases with medical procedure or neuron incitement. The vast majority of the individuals with epilepsy can be sans seizure with against seizure medicine. Not every one of the instances of epilepsy are long lasting and numerous individuals improve to the point of treatment. Epilepsy is extremely difficult to analyze and troublesome in finding the confusion [2].

Epilepsy can be regularly affirmed with an electroencephalogram (EEG). It is strategy for checking and recording electrical action of the brain. During the test terminals are set along the scalp. The typical example of mind waves will be changed, notwithstanding when an individual isn't having a seizure however an epilepsy. It is conceivable to analyze epileptic seizure by assessment of the recorded sign utilizing effective systems [3]. The fig. 1 (https://www.brainlatam.com/products/eegelectrode-caps) shows the EEG electrode placement

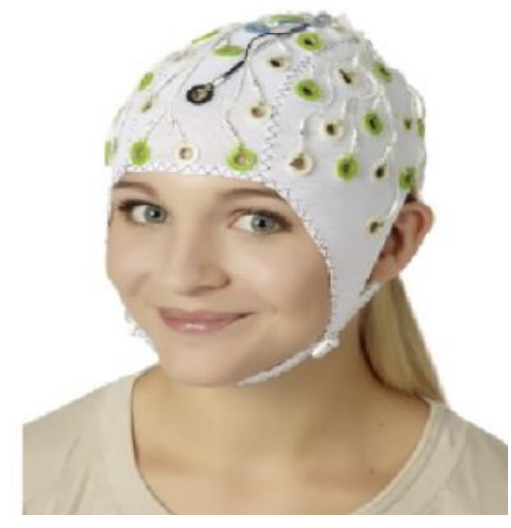

Fig. 1. EEG electrode placement

Epilepsy patients uncover two phases of strange exercises with their EEG signals i.e., ictal and interictal. Ictal is the record of an EEG while a seizure is happening (waveforms with sharp and spikes). Interictal is the period between the seizures (transient waveform i.e., spiky and sharp). By assessment of long term of EEG signals, experienced nervous system specialists with regular strategy uncovers epilepsy. In any case, this technique requires long term of time and helpless against analysis of blunders. Consequently Computer-helped 
location (CAD) of epileptic EEG sign can be used to vanquish these restrictions [4].

The electrical sign of brain waves has extremely little amplitude which is in the scope of 100 microvolts. The recurrence is in the middle of 0.44 $\mathrm{Hz}$ and $80 \mathrm{~Hz}$. Typically, EEG sign are arranged into five sub-groups, specifically delta $(0.5-4 \mathrm{~Hz})$, theta (4$8 \mathrm{~Hz})$, alpha $(8-12 \mathrm{~Hz})$, beta $(12-30 \mathrm{~Hz})$ and gamma $(30-60 \mathrm{~Hz})$. Table 1 demonstrates the amplitude and frequency for each kind of waves [5].

TABLE I. WAVE'S FREQUENCY AND AMPLITUDE

\begin{tabular}{lcc}
\hline \multicolumn{1}{c}{ Wave } & Frequency ranges & Amplitude \\
\hline Delta band & $0.5-4 \mathrm{~Hz}$ & High \\
Theta band & $4-8 \mathrm{~Hz}$ & Low-medium \\
Alpha band & $8-15 \mathrm{~Hz}$ & Low \\
Beta band & $15-30 \mathrm{~Hz}$ & Very low \\
Gamma band & $30-60 \mathrm{~Hz}$ & Smallest \\
\hline
\end{tabular}

In this paper, a new epilepsy identification technique based on VMD, RELIEFF, and machine learning classification approach is proposed. Variation mode decomposition (VMD) is a new adaptive signal decomposition technique which decomposes any real-time signal into band-limited functions or variation modes and extracts the features. The main purpose of feature extraction is to obtain the unique properties hidden in the EEG signals. The suggested method also employed a RELIEFF algorithm to choose the best features of the EEG signals. Finally, machine learning classification approach is used to classify the EEG signals.

The remainder of this paper is organized as follows. In Section II literature review of existing works are presented. Section III presents detail discussion about the used techniques (i.e, VMD, RELIEFF, ANN and KNN). With the extracted features performance of each classifier with respect to their confusion plots for finding performance parameters discussed in section IV. The obtained experimental results are also discussed in this section. Finally, concluding remark of the proposed method is presented in Section V.

\section{Review of Literature}

In this section, the most relevant work done in EEG signal processing, epilepsy detection and classification are discussed briefly.

Weng and Khorasani [6] proposed a versatile structure neural system (NN) with application to EEG seizure ditection in a computerized manner.

A technique proposed by Sunil and Harikumar [7] has utilized Singular Value Decomposition for a post classifier to arrange the epilepsy seizure level.
Utilizing Auto relapse models framed with SVM and PSO, the examination determination of epilepsy was done by Abdurrahaman et al [8].

Harikumar et al [9] performed comparison analysis between Particle Swarm Optimization (PSO) and Support Vector Machines (SVM) for easy epilepsy classification.

A method proposed by Juarez-Guerra et al. [10] to plan an epilepsy seizure identification utilizing EEG signals. In their procedure, to channel the antiques both finite impulse response (FIR) and infinite impulse response (IIR) were utilized, and after that to part the sign into five sub groups they utilized wavelet examination. At long last to extract features discrete wavelet transform (DWT) and maximal overlap discrete wavelet analysis (MODWT) were connected. By utilizing FeedForward Neural Network arrangement, they accomplishing an exactness of $93.23 \%$.

A strategy proposed by Dawood Dilber et al. [11] utilizing wavelet transform to extract the frequency domain and time domain features for grouping and recognition of epilepsy seizure. To separate epileptic and non-epileptic the proposed machine learning approach, for example, DAT and SVM and it accomplished an exactness of $70 \%$, and 93\% individually.

A technique was proposed by Md. Mamun o Rashid et al. [12] to split EEG signals DWT was used to extract statistical features from the signal. After that, the extracted features fed to a Neural Network machine learning to determine epileptic and nonepileptic signals.

\section{Proposed method}

The aim of this work is to develop an algorithm which can detect epilepsy based on the processing of EEG signals. The suggested method uses VMD, NCFS, and machine learning algorithms. In the next section, a brief explanation and underlying mathematical expression are provided for the used techniques.

\section{A. Variational Mode Decomposition}

Variational mode decomposition (VMD) is a new adaptive signal decomposition technique and it decomposes any real time signal into a band limited functions or variational modes $\left(u_{k}\right)$. Each mode occurred concurrently and exhibit the sparsity property for reconstruction of an input signal. VMD decomposes a real time signal into $\mathrm{k}$ modes $\left(u_{k}\right)$ around its center frequency $(\omega)$. Hilbert transform and frequency shifting property are useful parameters in formulation of an optimization problem. The formulation of a constrained variational problem as [13], 
$\min _{\left\{u_{k}\right\},\left\{\omega_{k}\right\}}\left\{\sum_{k}\left\|\partial_{t}\left[\left(\delta(t)+\frac{j}{\pi t}\right) * u_{k}(t)\right] e^{-j \omega_{k} t}\right\|_{2}^{2}\right\}$ (1)

$$
\sum_{k} u_{k}=f
$$

The quadratic penalty factor and Lagrangian multiplier $(\lambda)$ are converts into (2) from (1), The unconstrained optimization problem is expressed as (2);

$1\left(\left\{u_{k}\right\},\left\{\omega_{k}\right\}, \lambda\right):=\alpha \sum_{k} \| \partial_{t}\left[\left(\delta(t)+\frac{j}{\pi t}\right) * u_{k}(t)\right] e^{-j \omega_{k} t} \mid$

$\left\|f(t)-\sum_{k} u_{k}(t)\right\|_{2}^{2}+\left\langle\lambda(t), f(t)-\sum_{k} u_{k}(t)\right\rangle$

The alternate direction method of a multiplier (ADMM) is an optimization method to solve (2) lagrangian function 1 , it estimate modes around its own center frequencies. The wiener filter is embedded in a VMD to update each mode $u_{k}(\omega)$ optimally in a spectral domain.

The mode decomposition of normal and epilepsy EEG signals are in Figure 2a, Figure 2b shown respectively. It signifies that the mode increases also its frequency increases.
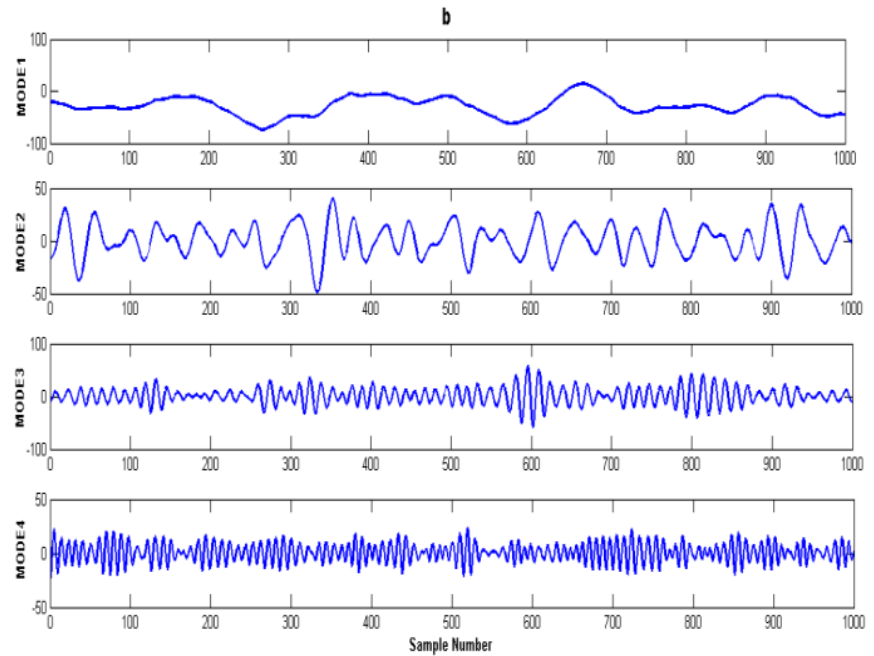

Fig. 2. VMD of four modes (a) Epilepsy signal (b) Normal EEG signal examples

\section{B. Feature extraction:}

\section{1) Statistical features:}

For an $\mathrm{N}$ sample EEG signal $y[n]$ gives the magnitude spectrum of $Y[\mathrm{~m}]$.

\section{Statistical features:}

Mean: It is the average of an $\mathrm{N}$ sample EEG signal; it can be defined [14],

$$
\mu=\frac{1}{N} \sum_{i=1}^{N} Y_{i}
$$

Standard deviation: The dispersion of data from it's a mean value of a signal is a standard deviation. It is derived as [14],

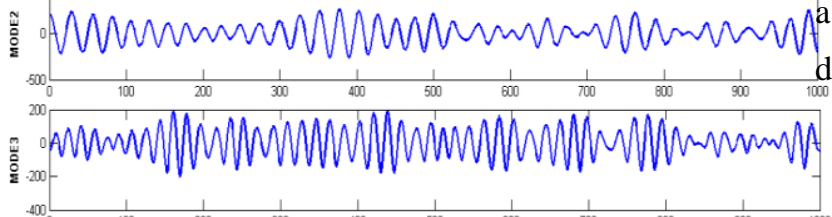

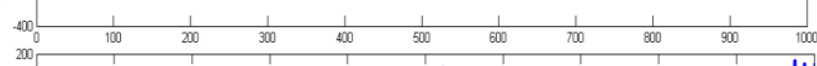

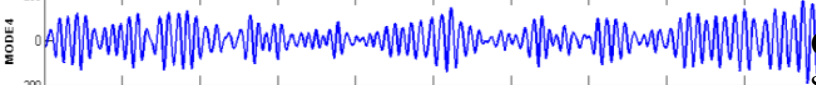

$$
\sigma=\sqrt{\frac{1}{N-1} \sum_{i=1}^{N}\left(Y_{i}-\mu\right)^{2}}
$$

(9)

Coefficient of variation (COV): The ratio of standard deviation to mean value of the EEG signal is a coefficient of variation. It can be expressed as [16].

$$
C O V=\frac{\sigma}{\mu}
$$

Entropy (H): It is defined as the measuring of randomness in EEG signals. For an EEG signals with $\mathrm{N}$ number of samples $\left(y_{1}, \mathrm{y}_{2}, \mathrm{y}_{3}, \ldots Y_{N}\right)$ is expressed as [14], 


$$
H(Y)=-\sum_{i=1}^{N} p\left(y_{i}\right) \log \left(p\left(y_{i}\right)\right) \quad p\left(y_{i}\right)=\left[p\left(y_{1}\right), p\left(y_{2}\right) \ldots . . .\right]
$$

Inter quartile range (IQR): It is defined as the difference between $75^{\text {th }}$ and $25^{\text {th }}$ percentile of samples, It measures variability in a data set is given by [15],

$$
I Q R=\mathrm{Q}_{3}-\mathrm{Q}_{1}
$$

Here, Q3 and Q1 are third and first quartile respectively.

Skewness: It measures the symmetry shape of the distribution of a signal, It can be derived as

$$
\text { Skewness }=\frac{1}{N} \sum_{i=1}^{N}\left(\frac{y_{i}-\mu}{\sigma}\right)^{3}
$$

NegEntropy: The differences between gaussian entropy $\mathrm{H}($ Ygauss) and differential entropy $\mathrm{H}(\mathrm{Y})$ having mean $\mu$ and variance $\sigma^{2}$ of EEG signals. It is expressed as [14],

$$
J(Y)=H\left(Y_{\text {gauss }}\right)-H(Y) \quad H\left(Y_{\text {gauss }}\right)=\frac{1}{2} \log \left(2 \pi e \sigma^{2}\right)
$$

Kurtosis: It is a one of the statistical moment; it gives the time series data peaked nature. Kurtosis can be derived as [17]

$$
k=\frac{1}{N} \sum_{i=1}^{N}\left(\frac{y_{i}-\mu}{\sigma}\right)^{4}
$$

\section{2) Spectral features:}

Spectral flatness (SF): Spectral flatness can be define as the ratio of magnitude spectrum of geometric mean to the arithmetic mean, It can be expressed as $[14,16]$,

$$
S F=\frac{\prod_{m=0}^{N-1}|Y[m]|^{\frac{1}{N}}}{\frac{1}{N} \sum_{m=0}^{N-1}|Y[m]|}
$$

Spectral spread (SS): Average deviation of a magnitude spectrum around its spectral centroid is called as spectral spread. It can also be assumed as instantaneous bandwidth, It can be mathematically expressed as [14,16],

$$
S S=\frac{\sum_{m=0}^{N-1}(m-S C)^{2}|Y[m]|}{\sum_{m=0}^{N-1}|Y[m]|}
$$

It is a ratio of sum of a weighted magnitude spectrum to normalized by an unweighted sum is called as spectral centroid (SC),

$$
S C=\frac{\sum_{m=0}^{N-1} m|Y[m]|}{\sum_{m=0}^{N-1}|Y[m]|}
$$

Spectral decrease (SDec): It measuring the amount of decrease of a spectral envelope of a signal with respect to frequency, it is denoted as [14],

$$
\text { SDec }=\frac{\sum_{m=1}^{N-1} \frac{1}{m} \cdot(|Y[m]|-|Y[0]|)}{\sum_{m=1}^{N-1}|Y[m]|}
$$

\section{C. $\quad K$-Nearest Neighbor Classifier $(K N N)$ :}

$\mathrm{KNN}$ is one of the finest learning algorithm, until the classification whole calculation differs in which function approximated locally only. KNN algorithm is one of the simplest Machine Learning algorithms. In order to catalogue the unknown instance with class label as that of known neighbor and to locate the nearest neighbor in instance set. Classification with an instant classifier can be straight forward task. Class membership is the output in KNN classification to classify, the training and testing datasets of epileptic EEG classification are applied to KNN and the documentation of classification result is done.

\section{Artificial Neural Network (ANN):}

In machine learning and cognitive science a family of statistical learning models are said to be artificial neural networks and are inspired by biological neural networks. Using machine learning process, the training dataset is used to train ANN, for epileptic seizure classification using ANN the test EEG dataset is used for testing the performance.

\section{E. RELIEFF}

Relieff is an algorithm which is popularly used for filtering feature selection in a very effective manner. It has high end application to binary classification problems with discrete or numerical features. Inductive machine learning problems typically use greedy search, however we use Relief algorithm for heuristic guidance of inductive learning algorithms. 
The basic idea of RELIEFF is to estimate attributes conferring to how well their values differentiate among the instances that are near to each other. For given an instance, RELIEFF searches for its two nearest neighbors: one from the same class called nearest hit (denoted by $\mathrm{H}$ ) and the other from the different class called nearest miss (denoted by M). The original algorithm of RELIEFF [18] [19] randomly selects $n$ training instances, where $n$ is the user-defined parameter. The algorithm is as fallows.

1. set all weights $\mathrm{W}[\mathrm{A}]=0.0$;

2. for $\mathrm{i}=1: \mathrm{n}$

3. randomly select an instance $\mathrm{R}$;

4. find nearest hit $\mathrm{H}$ and nearest miss $\mathrm{M}$;

5. for $\mathrm{A}=1$ : \#all_attributes

6. $\mathrm{W}[\mathrm{A}]=\mathrm{W}[\mathrm{A}]-\operatorname{diff}(\mathrm{A}, \mathrm{R}, \mathrm{H}) / \mathrm{n}$

7. $+\operatorname{diff}(\mathrm{A}, \mathrm{R}, \mathrm{M}) / \mathrm{n}$;

8. end;

9. end;

The weights $\mathrm{W}[\mathrm{A}]$ estimates the quality of attributes. The basis of the formula for updating the weights is that a good attribute should have the same value for instances from the same class (subtracting the difference $\operatorname{diff}(\mathrm{A} ; \mathrm{R} ; \mathrm{H})$ ) and should differentiate between instances from different classes (adding the difference diff (A; R; M)).

The

function diff(Attribute,Instance1,Instance2) calculates the difference between the values of Attribute for two instances instance1 and instance2. For discrete attributes the difference is either 1 (the values are different) or 0 (the values are equal), while for continuous attributes the difference is the actual difference normalized to the interval $[0 ; 1]$. Normalization with $\mathrm{n}$ guarantees all weights W[A] to be in the interval $[-1 ; 1]$, however, normalization with $\mathrm{n}$ is not required if W[A] is to be used for relative comparison among attributes.

The total distance is simply the sum of differences of all attributes. In fact original RELIEFF uses the squared difference, which for discrete attributes is equivalent to diff. In all the experiments, there was no substantial difference between results using diff or squared difference. If $\mathrm{N}$ is the number of all training instances then the complexity of the above algorithm is $\mathrm{O}$ ( $\mathrm{n} \times \mathrm{N}$ x \#all_attributes).

\section{Results and Discussion}

In this paper, an efficient method based on VMD, RELIEFF and machine learning approach is designed, developed and implemented for classification of the epileptic seizures. Matlab platform has been used to implement and simulate the proposed classification algorithm and the achieved simulation results are presented in this section. The EEG dataset is adopted from the Bonn University Hospital of Freiburg [20]. It contains five individual subsets (set A-E) named as Z,O,N,F and S. Each subset consists 23.6s duration of 100 single channel EEG signals. The data has a sampling rate of 173.61 $\mathrm{Hz}$ and digitalized with 12-bit analog to digital resolution. Set $\mathrm{A}$ and set $\mathrm{B}$ are captured extra cranially whereas remaining collected intracranial with a standardized 10-20 electrode system. Set A was recorded from the healthy patients when eyes open, set B was recorded from the healthy patients when eyes close, Set $C$ and set D were recorded when patients are in seizure free intervals, Set E consists of epileptic seizure signals and these signals show ictal activity.

To evaluate the proposed methods performance, accuracy, sensitivity, specificity, precision, F_measure, and G_mean classifier performance parameters are used.

$$
\begin{array}{r}
\text { Accuracy }=\frac{T P+T N}{(T P+F N)+(T N+F P)} \\
\text { Sensitivity }=\frac{T P}{(T P+F N)}
\end{array}
$$

$$
\text { Specificity }=\frac{T N}{T N+F P}
$$

$$
\text { Precesion }=\frac{T P}{T P+F P}
$$

$$
F_{-} \text {measure }=\frac{2(\operatorname{Precesion} \times \text { Sensitivit })}{(\operatorname{Pr} e c e s i o n+\text { Sensitivity })}
$$

$$
G_{-} \text {mean }=\sqrt{(\text { Specificity } \times \text { Sensitivity })}
$$

The obtained simulation results of an epileptic seizure classification algorithm using ANN are presented in Table 2.

TABLE II. CONFUSION MATRIX OF EPILEPTIC SEIZURE CLASSIFICATION USING ANN WITH RELIEFF

\begin{tabular}{ccc}
\hline Signal & Epilipsy(\%) & Normal $(\%)$ \\
\hline Epilipsy & 97.5 & 2.5 \\
Normal & 11.7 & 88.3 \\
\hline
\end{tabular}


From the obtained confusion matrix, it can be observed that the classification algorithm using ANN with RELIEFF achieves an overall accuracy of $92.91 \%$, overall sensitivity of $97.50 \%$, overall specificity of $88.33 \%$, overall precision of $89.31 \%$, overall F_measure of $93.22 \%$ and overall G_mean $92.80 \%$.

Simulation results of an epileptic seizure classification algorithm using KNN with RELIEFF are presented as follows in Table 3. According to the confusion matrix obtained, $\mathrm{t}$ is observed that the classification algorithm using KNN with FMFS achieves an overall accuracy of $93.33 \%$, overall sensitivity of $96.67 \%$, overall specificity of $90.00 \%$, overall precision of $90.62 \%$, overall F_measure of 93.54\% and overall G_mean $93.27 \%$.

TABLE III. CONFUSION MATRIX OF EPILEPTIC CLASSIFICATION USING KNN WITH RELIEFF

\begin{tabular}{ccc}
\hline Signal & Epilipsy(\%) & Normal(\%) \\
\hline Epilipsy & 96.7 & 3.3 \\
Normal & 10.0 & 90.0 \\
\hline
\end{tabular}

TABLE IV. PERFORMANCE SUMMARY OF AN EPILEPTIC SEIZURE CLASSIFICATION

\begin{tabular}{llll}
\hline S.No & Parameter & ANN+RELIEFF & KNN+RELIEFF \\
\hline 1 & Accuracy & 92.91 & 93.33 \\
2 & Sensitivity & 97.50 & 96.67 \\
3 & Specificity & 88.33 & 90.00 \\
4 & Precision & 89.31 & 90.62 \\
5 & F_measure & 93.22 & 93.54 \\
6 & G_mean & 92.80 & 93.27
\end{tabular}

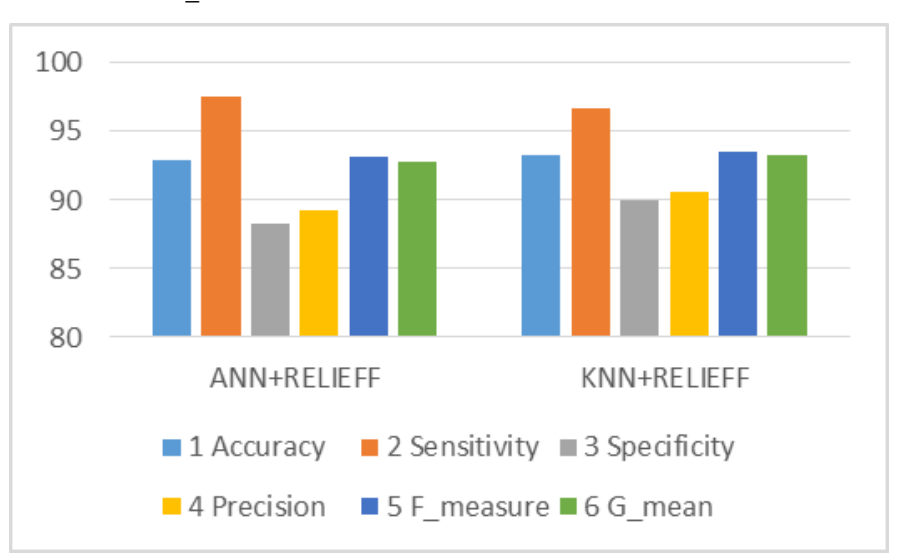

Fig. 3. Bar graph comparison of performance parameters

TABLE V. COMPARISON TABLE

\begin{tabular}{|l|c|c|c|}
\hline \multicolumn{1}{|c|}{ Title } & Author & Method & Results \\
\hline $\begin{array}{l}\text { Performance Analysis of SVD and K-means } \\
\text { Clustering for Optimization of Fuzzy outputs in } \\
\text { Classification of Epilepsy Risk Level from EEG } \\
\text { Signals[7] }\end{array}$ & $\begin{array}{c}\text { Dr. } \\
\text { R.Harikumar }\end{array}$ & k-clusteer+fuzzy & 92.79 \\
\hline $\begin{array}{l}\text { Regularization And Kernel Parameters } \\
\text { Optimization Based On PSO Algorithm In EEG } \\
\text { Signals Classification With SVM[8] }\end{array}$ & Abdurrahaman & PSO+SVM & $82.75($ linear) \\
& $\begin{array}{c}91.00 \\
\text { (polynaamial) }\end{array}$ \\
\hline $\begin{array}{l}\text { Proposed Method } \\
\text { Kk Ebraheem } \\
\text { Khaleelulla }\end{array}$ & VMD+RELIEFF+ & 93.33 \\
\hline
\end{tabular}

\section{Conclusion}

In this paper, methods for detection of epileptic seizure in EEG signals have been discussed. The adopted method used VMD for feature extraction and the obtained features are passed to RELIEFF algorithm for the selection of best features. The machine learning techniques namely, ANN and KNN are employed to in this work for the detection of epilepsy. The ANN+RELIEFF achieved 92.08\% accuracy and KNN+RELIEFF achieved $93.33 \%$ of accuracy. From the overall obtained result it can be concluded that KNN, RELIEFF with VMD is able to provide highly improved performance.

\section{References}

[1] Z. Lasefr, Sai Shiva V N R Ayyalasomayajula, and K. Elleithy, "An efficient automated technique for epilepsy seizure detection using EEG signals," In Proc. IEEE 8th Annual Ubiquitous Computing, Electronics and Mobile
Communication Conference (UEMCON), pp. 76-82, 2017.

[2] Acharya, U. Rajendra, et al. "Application of entropies for automated diagnosis of epilepsy using EEG signals: a review." Knowledge-Based Systems 88 (2015): 85-96.

[3] Kalaivani, M., V. Kalaivani, and V. Anusuya Devi. "Analysis of EEG Signal for the Detection of Brain Abnorrnalities." JJCA Proceedings on International Conference on Simulations in Computing Nexus. No. 2. Foundation of Computer Science (FCS), 2014.

[4] SK. Ebraheem Khaleelulla and Dr. P. Rajesh Kumar, "a novel statistical approach for detection and classification of epileptic seizures using DWT and SVM, " International Journal of Current Engineering and Scientific Research (IJCESR), Vol. 5, No. 3, pp. 1-7, 2018.

[5] Ebraheem Khaleelulla S., Rajesh Kumar P. (2018) EEG Signal Analysis for Mental States and Conditions of Human Brain. In: Satapathy S., Bhateja V., Chowdary P., Chakravarthy V., Anguera J. (eds) Proceedings of 2nd International Conference on Micro-Electronics, 
Electromagnetics and Telecommunications. Lecture Notes in Electrical Engineering, vol 434. Springer, Singapore

[6] Weng, W., \& Khorasani, K. (1996). An Adaptive Structure Neural Networks with Application to EEG Automatic Seizure Detection. Neural Networks, 9(7), 1223-1240. doi:10.1016/08936080(96)00032-9

[7] P, S. K., \& Rajaguru, H. (2016). Assessment Of Epilepsy Classification Using Techniques Such As Singular Value Decomposition, Approximate Entropy, And Weighted K-Nearest Neighbors Measures. Asian Journal of Pharmaceutical and Clinical Research, 9(5), 91. doi:10.22159/ajpcr.2016.v9i5.12196

[8] Abdurrahaman, Ismail $\mathrm{M}$ and Ramzan "Regularization And Kernel Parameters Optimization Based On PSO Algorithm In EEG Signals Classification With SVM", 2011 IEEE 19th Signal Processing and Communications Applications Conference (SIU 2011):399-402

[9] Hari kumar rajaraju and Suneel kumar p "Comparison Of Support Vector Machine With Particle Swarm Optimization Technique For Epilepsy Classification From Eeg", International Journal Of Pharmacy \& Technology, ISSN 0975-766X

[10] E. Juarez-Guerra, V. Alarcon-Aquino, and P. Gomez-Gil, "Epilepsy seizure detection in EEG signals using wavelet transforms and neural networks," in New Trends in Networking, Computing, E-learning, Systems Sciences, and Engineering, ed: Springer, 2015, pp. 261-269.

[11] D. Dilber and J. Kaur, "EEG based detection of epilepsy by a mixed design approach," in 2016 IEEE International Conference on Recent Trends in Electronics, Information \& Communication Technology (RTEICT), 2016, pp. 1425-1428.

[12] M. M. o. Rashid and M. Ahmad, "Epileptic seizure classification using statistical features of
EEG signal," in 2017 International Conference onElectrical, Computer and Communication Engineering (ECCE), 2017, pp. 308-312.

[13] Dragomiretskiy K, Zosso D. "Variational mode decomposition". IEEE Trans Signal process. 2014;62(3):531-44

[14] LerchA."An introduction to audio content analysis: applications in signal processing and music informatics". Hoboken: Wiley; 2008

[15] Taran S, Bajaj V, Sharma D. Robust "Hermite decomposition algorithm for classifcation of sleep apnea EEG signals". Electron Lett. 2017;53(17):1182-4

[16] Hassan AR, Haque MA. "Computer-aided obstructive sleep apnea screening from singlelead electrocardiogram using statistical and spectral features and bootstrap aggregating". Biocybern Biomed Eng. 2016;36(1):256-66.

[17] R. Panda, P. Khobragade, P. Jambhule, S. Jengthe, P. Pal, and T. Gandhi, "Classification of EEG signal using wavelet transform and support vector machine for epileptic seizure diction," in Systems in Medicine and Biology (ICSMB), 2010 International Conference on, 2010, pp. 405-408.

[18] K. Kira and L. Rendell.," A practical approach to fea-ture selection". Proc. Intern. Conf. on Machine Learn-ing (Aberdeen, July 1992) D.Sleeman and P.Edwards

[19] K. Kira and L. Rendell., "The feature selection prob-lem: traditional methods and new algorithm”. Proc.AAAI'92, San Jose, CA, July 1992.

(eds.), Morgan Kaufmann, pp.249-256.

[20] R. G. Andrzejak et al., "Indications of nonlinear deterministic and finite-dimensional structures in time series of brain electrical activity: dependence on recording region and brain state," Phys. Rev. E, vol. 64, no. 6, pp. 116-126, Nov. 2001. 